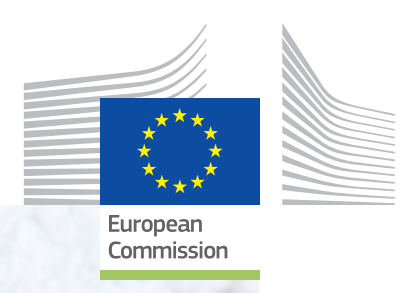

\title{
State of Paediatric Medicines in the EU
}

10 years of the EU Paediatric Regulation

Report from the Commission to the European Parliament and the Council

COM (2017) 626 
State of Paediatric Medicines in the EU

10 years of the EU

Paediatric Regulation

Report from the Commission to the European Parliament and the Council 
1.

\section{Introduction}

Nowadays there is broad consensus that children deserve access to medicines that have been specifically developed and researched for their use in young patients. However, until recently the development and testing of paediatric medicines was far from salisfactory. Many of the products used in childen wer pescrbed and administered based on physcians own based on physicians' own experience rather than on the results of clinical research. Moreover, medicines were often not available in a pharmaceutical form suitable to children. Paediatricians had to turn to medicines authorised for adults by adapting the dosage and form. For example by crushing adult tablets and using only a portion. This off-label use of adult medicines comes with the risk of inefficacy and/or adverse reactions in children. Side effects that may not affect adults can be important and serious in children.

Surveys suggested that in many therapeutic areas the off-label use was widespread, often reaching figures above $50 \%$ Childhood immunisation was a notable exemption, being one of the success stories of modern medicine.

There are several reasons why paediatric medicine development had been largely neglected. Until the 1980s it was often argued that children should be protected from clinical research for ethical reasons. Since then there has been a gradual shift to the current consensus that children merit the same level of health care as any other age group including evidencebased prescribing of medicinal products. based prescibing of medicinal products. Economic considerations were also a contributing factor as to why companies re frained from proactively investing in this sector. The fact that children grow and maturate means that they are not a uniform sub-group. The needs and biological and physiological characteristics of different compared to teenagers. Therefore additional age-ap- propriate research is often needed, mak ing the process of developing paediatric medicines more complex.

The Paediatric Regulation' ('the Regulation') was adopted to address this problem. Legislative intervention was deemed necessary to reverse previous trends. A consultation and discussion process that consultation and discussion process that lasted several years was the basis of the legislation. It was also inspired by developments in the United States, which started legislative approaches to address paediatric product development in the late 1990s.

2017 marks the 10th anniversary of the Regulation. In line with its Article 50(3), this report provides an account of its achievements, both in public health and economic terms and an analysis on the extent to which its objectives have been met While 10 years provides a rich database of experience, it remains a relatively short period of time in view of the long short period of time in view of the long development cycles of medinal products, amounting often close to a decade.

This report builds on a 10-year report prepared by the European Medicines Agency (EMA) and its Paediatric Committee ${ }^{2}$ an ordered by the Comission's impact ordered by the Commission ${ }^{3}$, a public consultation and discussions with Member States, the European Parliament ${ }^{4}$, patients, companies, interested parties and external partners about their experiences on the Regulation's impact.

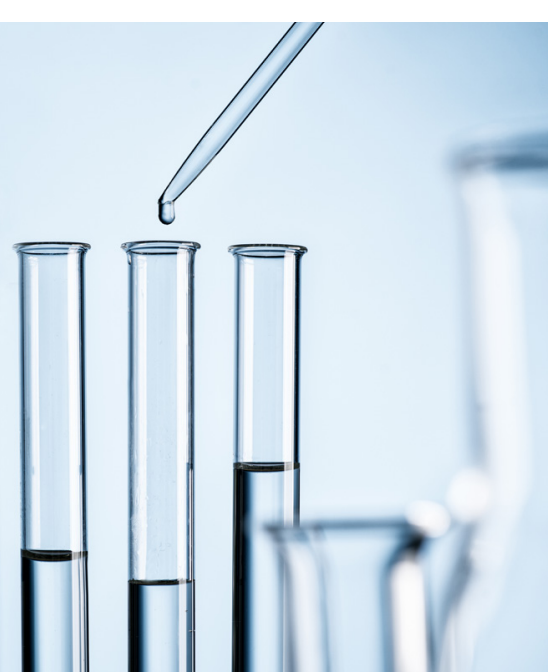

1. Regulation (EC) No

European Parliament and of the
Council of 12 December

2006 on medicinal products

for paediatric use, OJ L 378 , 27.12.2006, p. 1 .

2. 10-year report to the

- General report on the

experience acquired as a

result of the application of

the Paediatric Regulation

3. Technopolis, Study on

the economic impact of

including its rewards and

incentives, 2017

4. European Parliament
resolution of 15 December

2016 on the regulation on

paediatric medicines. 
5. Regulation (EC) No Parliament and of the Corncil of 16 December 1999 o orphan medicinal products

6. Regulation (EC) No 469/2009 of the Eurothe Councili pf 6 May 2009 concerning the supplementary protection certificate for medicinal products, OJ L 152 16.6.2009, p. 152

\section{2.}

The Paediatric

\section{Regulation}

The Regulation is structured around three main objectives:

\section{- to encourage and enable}

high-quality research into the development of medicines for children;

to ensure, over time, that most medicines used by chitdren are specifically authorised for such use with age-appropriate form use with age appropriate forms and formulations; and

to increase the availability of high-quality information about medicines used by children.

To meet these objectives the Regulation sets up a system of obligations, rewards and incentives, and puts in place mards and incentives, and measures to e regularly researched, developed and authorised to meet children's therapeutic needs. It is based on the simple idea that a company should be obliged to screen every product it develops for its potential use in children, thereby progressively increasing the number of

The Regulation obliges companies to agree at an early stage of development a paediatric Research and Development programme ('paediatric investigation plan') with the EMA. The Regulation has a direct impact on companies' R\&D expenditure, as it imposes an investment in paediatric research. If a company fails to comply with the agreement, the respective (adult) marketing authorisation may be blocked. The Regulation therefore goes beyond the mechanisms set up by the legislation on medicines for rare diseases ('Orphan Regulation') 5 , which only provides incentives for companies.
The obligation laid down in the Regulation is complemented by other measures, in particular:

$\begin{array}{ll}\text { - a system of waivers for medi- } & \text { basically forced to establish paediatric } \\ \text { cines that are unlikely to benefit } & \text { infrastructure and to build expertise to } \\ \text { children and a system of defer- } & \text { ensure appropriate paediatric research } \\ \text { cals in relation to the timing of } & \text { velopment. }\end{array}$

rals in relation to the timing of the paediatric measures to be conducted;

a reward for complying with the obligation: a six-month extension of the supplementary protection certificate;

a specific reward for orphan medicines: an extra two years of market exclusivity added to the existing 10 years awarded under the Orphan Regulation;

a new type of marketing authorisation, the paediatric use marketing authorisation (PUMA), the development of paediatric indications for offpatent products;

an expert committee, the Paedi- atric Committee (PDCO), within EMA; and

- a system of free scientific advice for the industry, provided by EMA.

In addition, the Regulation promotes high-quality information and high-quality research through other measures, such as

- an EU network of networks of investigators and trial centres carrying out paediatric research (Enpr-EMA);

- an EU inventory of paediatric needs;

a public database of paediatric studies; and

- a requirement for companies to submit any existing paediatric studies on authorised medicinal products for scrutiny by regulatory authorities.
One of the Regulation's undisputed achievements is bringing more attention and financial investment to paediatric development. Companies were velopment.

In 2013, the Commission published first report on the Regulation's impact and concluded that there are some promising signs of progress ${ }^{7}$. Howeve it found that, because of the length of medicinal products' development, it will take at least 10 years to gain a full understanding of the situation.

Article 50(3) of the Regulation requires the Commission to publish a second report in 2017. The second report should also consider whether amendments to the Regulation should be contemplated.

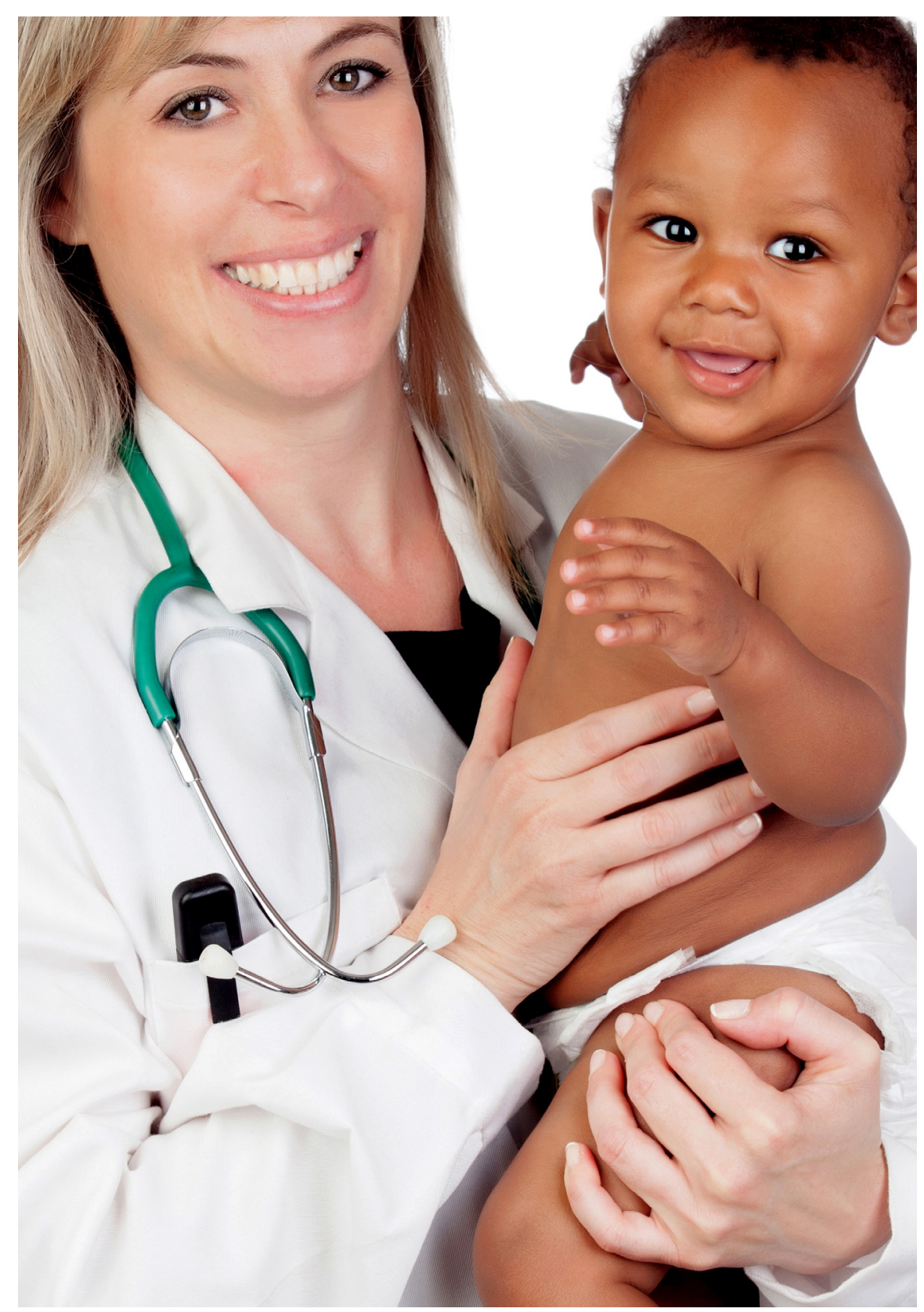

Better Medicines for From concept to 


\section{MORE}

MEDICINES

FOR CHILDREN

Figures show that the Regulation has had a substantial impact on the development of paediatric medicines in the EU. Pharmaceutical companies now consider paediatric development as an integral part of the overall development of medicinal products, even if some of them continue to perceive paediatric research as regulatory-driven rather than company-driven.

In 2007-2016 over 260 new medicines for use by children (new marketing authorisations and new indications) were authorised, most of them linked to the Regulation's requirements. The number of agreed paediatic investigatior ber of agreed paediatric investigation plans (PIPs) surpassed 1000 in 2017, of which 131 were completed at the end of 2016. There is a clear upward trend in the number of completed PIPs, with over $60 \%$ finalised in the last three years. In addition, competent authorities' assessments of paediatric studies undertaken mere the Regulation (Article 45) have before helped to consolidate already existing evidence and to complement product information with paediatric data.

A comparison of the situation before and after the Regulation demonstrates a clear positive effect in terms of new a clear postive authorised medines. The same is true for international-level comparisons between legal systems with paediatricspecific legislation and those without: legal systems with legislative provisions in place have a significantly higher number of new paediatric medicines.

The above quantitative analysis shows clear progress. Those output figures are also in line with expectations taking into account that bringing a medicinal product to the market may take up to 10 years, underlining the incremental change the Regulation provides.
At the same time, issuing a marketing authorisation or adding paediatric information to existing marketing authorisations does not automatically translate into the immediate availability of the product to all paediatric patients in the pr. This may be due to panding reime to bending reimbursement decisions at national level or prescription habits, where physicians may not directly switch to newly authorised products. In response to a survey that provided input to this report ${ }^{8}$, the majority of respondents estimated that the increase in available medicines was in the range of 5-10\%. On prescribing habits, $58 \%$ of respondents indicated that practitioners are increasingly prescribing approved medicines according to their licensed indication for children, as a result of the Regulation. This demonstrates a positive trend, but also underlines certain inertia. The reduction of off-label use in children is finally not only dependent on more authorised paediatric medicines, but on real availability and use at bed-side.

In this context, it is observed that companies often rely on a staggered roll-out of new products with the consequence of delays until the product is finally available throughout the EU. This cannot be fully prevented even if the Regulation includes several instruments to ensure that, once a PIP is completed and the paediatric medicine is authorised, the product is placed on the market. For example, the supplementary protection certificate reward under Arthe product is authorised in all Member States. Article 33 also contains an obligation to place the product on the market within two years of the date on which a new paediatric indication is authorised.

The timely availability of paediatric medicines may also be impacted by the delayed completion of the paediatric studies compared to the finalisation and authorisation of the corresponding adult product. The Regulation includes provisions for deferring the initiation or completion of some or all measures contained in a PIP (Article 20) so as to ensure that research is carried out only when safe and ethical. Additionally, it is meant to avoid blocking or delaying the authorisation of products for the adult population.

Experience shows that the deferral is a widely used instrument. In practice nearly all PIPs for new medicines that are linked to an adult development include a to an adult development in ures erral is in principle a usefu prate instrument and there is no evidence that the paediatric requirements have delayed the processing of adult applications. However, the Paediatric Committee agreed in some cases to very long deferrals. This may cause frustration among clinicians and patients, especially if it means that a promising paediatric product will only be available years after the adult authoriMoreover, if the start of a paediatric trial is delayed until after the adult authorisation, experience shows that recruitment of patients in paediatric trials becomes more difficult Parents may fail to see the added value of agreeing that their child participates in clinical research if the adult product can already be used (off-label) in chilalso linked to the late submission of a PIP. While there is a downward trend in late submissions (currently 10-20\%), the agreement for deferral in those cases may require closer scrutiny to avoid that those late submissions come at the expense of swift progress in paediatric therapies.

Against this background, the EMA and its Paediatric Committee are currently reviewing past practices to ensure consistency and to avoid significant deferrals. In view of evolving science, it may be argued that agreeing to long therapeutic benefit development over existing treatments for paediatric patients. In such cases, the added-value of the paediatric studies might be marginal. Furthermore, long deferrals may un- dermine the enforceability of paediatric requirements, and the availability of any reward, especially if the deferral ends after the protection periods for the product have expired.

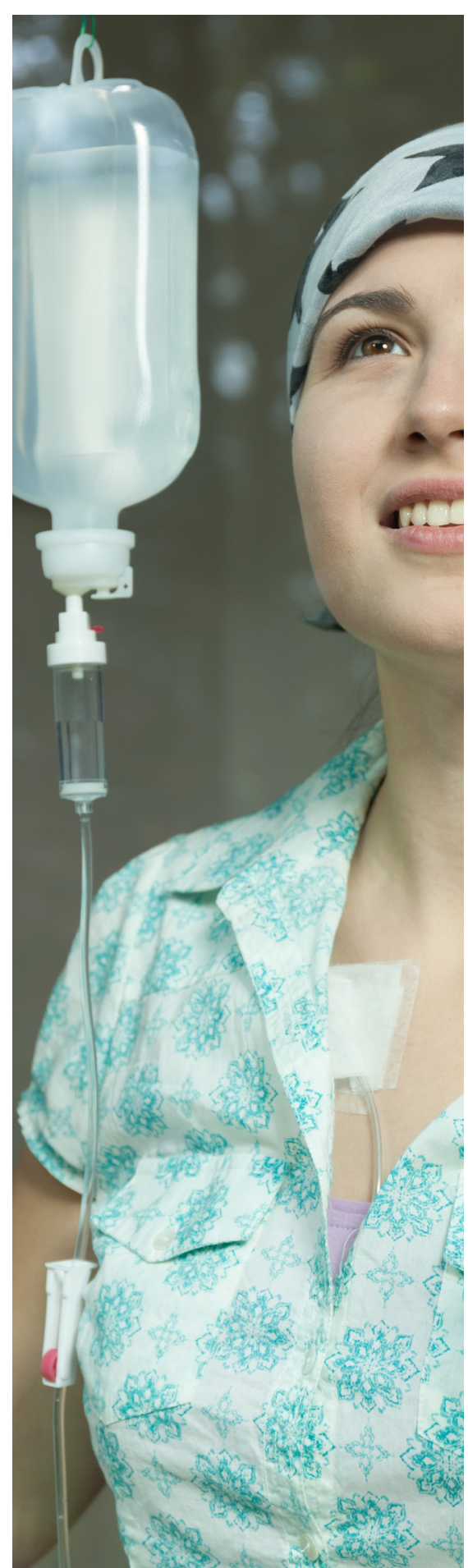




\section{BETTER \\ MEDICINES \\ FOR CHILDREN}

The last 10 years have seen some considerable progress in the availability of medicines for children in certain therapeutic fields because of the Regulation. Rheumatology or infectious diseases are often referred to as prime examples. The significant surge of new treatments for children with theumatologic diseases children with theumatologic diseases following the completion of PIPs has transformed a sector, which was previously neglected.

At the same time, those positive developments do not follow a strategic plan, but are often linked to developments in adult markets. As the starting point for adult markets. As the stanting point for most programme for adults, progress in a paediatric field is dependent on companies' adult product pipeline and influenced by revenue prospects in a specific market segment. Where the adult needs or market expectations overlap with paediatric needs, children will benefit directly. However, there a a considerable number of diseases that are biologically different in adults and children, where the disease burden differs, or that only exist in children. It is in those diseases, where the mechanism introduced by the Regulation sometimes struggles with scientific, clinical and market realities.

This cuts both ways. A recent example where a wave of new adult developments may risk overloading the system is type II diabetes; a disease that is marked by its ever growing prevalence among adults since the 1980s. Over the past years, many companies concentraten this therapeutic area leading to a ed on this theraptic area leading to a peak in activilies and an evolving pipeline of new products. Such waves lead in parallel to an increase in paediatric research programmes, even if - when seen from the perspective of therapeutic needs - having fewer of them might have been sufficient, given that type II diabetes is still relatively rare in children. The mismatch between disease burden at adult level and in children may also lead to feasibility problems on conducting paediatric trials, as there may be simply not enough young patients to be simply not enough young patients to be enrolled in PIP studies. To overcome such problems it has been suggested that companies should engage in collaborative research to make better use of the limited patient pool. However, companies are hesitant, especially if it concerns developments with potential blockbuster status in adults. At the same time, the Pactiatric Committee is not in a position Paedian Comnittee is not in a position to prioritise between PlPs for the same therapeutic area. It is often a 'catch-22' situation, as only the results of clinical trials could inform the Paediatric Committee in its choice of which compounds may provide the most promising results in children. However, the intervention of the Paediatric Committee and the agreement of a paediatric investigation plan usually take place before those results are available, as the purpose of a PIP is to identify and agree the studies that need to be conducted.

At the other end of the scale are diseases that are unique to the paediatric population, where paediatric development depends typically on the strategic decision of a company to invest in this area independently of any on-going adult programme. This is particularly true for rare diseases in children, such as paediatric cancer.

Reassuringly, an analysis of the agreed PIPs shows that they cover a wide range of therapeutic areas, with infectious diseases $(12 \%)$, oncology $(10 \%)$ and endocrinology/metabolic diseases (9\%) at the forefront, but no particular area dominates. Overall this is a good sign as it demonstrates paediatric activities covering a wide range of diseases. How number of agreed PIPs does not automatically mean a high number of completed PIPs. Currently, the conditions with the highest number of completed PIPs are immunology/rheumatology $(14 \%)$, infectious diseases (14\%), car- diovascular diseases and vaccines (each $10 \%$ ), with oncology and endocrinology/ metabolic diseases only corresponding to $7 \%$ of the completed PIPs. Moreover, the development in terms of agreed and completed PIPs does not necessarily correspond with the paediatric disease burden, wh ease burden, which underlines the fact that paediatric medicine development is often driven by adult development. The possibilities of the Regulation to steer activities towards certain therapeutic areas are limited. It is an important enabler, but the qualitative impact is still dependent on market forces, drivers of considerations of companies.

In the discussion on paediatric needs paediatric oncology is often used as a case study for insufficient advances in an area of high unmet paediatric need.

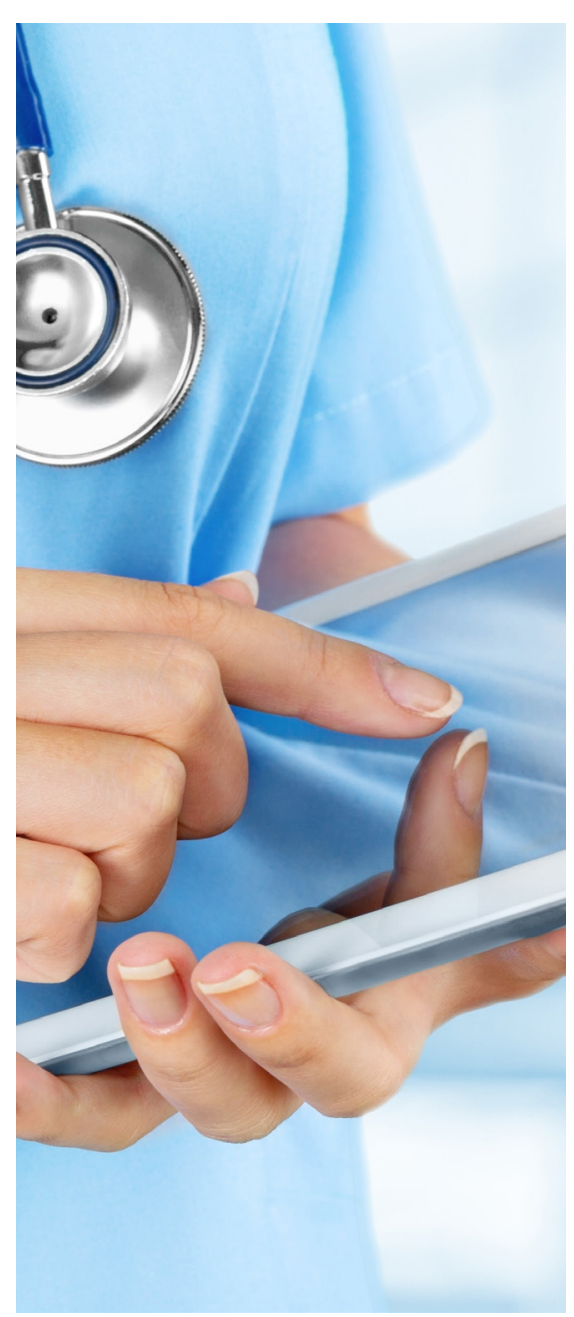

Although cancer in children is rare, it is still the leading cause of death by dis ease past infancy despite improved survival rates for some types of cancer in recent decades.

The discussion on paediatric oncology is often linked to the waiver concept set out in Article 11 of the Regulation, which provides that the requirement for a PIP may be waived for specific products o classes of products under specific circumstances. This happens if a product is likely to be ineffective or unsafe for children or it does not have a significant therapeutic benefit over existing treatments. The obligation is also waived if the disease or condition for which the product is intended occurs only in adults.

The waiver aims to avoid unnecessary or even unethical research and to correctly frame the scope of obligations and is considered as an appropriate instrument In 2007-2016, EMA ganted some class In 2007-2016, EMA granted some class waivers and 486 product-specific waivers for use of a medicine in one or more conditions. However, while it is generally appropriate to waive paediatric studies, if the target disease does not exist in children, it is not excluded that the compound may still be beneficial for children, albeit in a differ ple, while many paediatric cancers share biological similarities with adult cancers, they occur in different organs and are therefore usually considered as different conditions. Consequently, a company may be entitled to a waiver even if the mechanism of action of the compound developed for adults and its molecula target may also be effective in treating certain paediatric cancers.

The last few years has seen a surge of innovative adult cancer drugs entering the market with some first-in-class products, creating better treatment options and improved patient outcomes and longer survival rates. Currently, cancer treatments represent the largest category of new medicines, also in view of their revenue potential. And it is expected that they will continue to transform the therapeutic landscape 
Roughly a quarter of all medicines currently in late development stage are cancer therapies. One indicator for the continued high interest in cancer drug development is also the Orphan Regulation, where cancer therapies belong to the most frequently designated orphan conditions revealing a trend towards narrowly focussed medicines ${ }^{10}$. However, this pace of advances observed in adult therapies is so far not mirrored in paediatric patients. In some paediatric cancers, the most used medicines date back to the 1990 s, if they exist at all.

At the same time, the Regulation has had an impact and has led to new anticancer products being authorised. Seven developments in PIPs have been completed, providing treatment options for high-grade glioma, rhabdomyosarcoma, astrocytoma and acute lymphoblastic leukaemia.

The number of agreed PIPs for anti-cancer medicines (68) covering more than 30 different mechanisms of action are a promise for further improvements in the future. Some of those PIPs are based on the mechanism of action principle, i.e. while the company could have potentially relied on a waiver it committed to pae diatric research in view of the potential diatric research in view of the benefit of the compound to treat childhood cancers.

A contributing factor to those commitments may have been secondary effects of the Regulation, which by definition encouraged companies to strengthen their expertise in paediatic expertise in paediatic drug development. This may have impacted strategic decisions of companies in favour of covering paediatric needs, in particular through the use of innovative trial designs, such as basket trials, by which a compound is tested against multiple cancer types to inform an early selection of the most promising developments. Moreover, the promising developments EU provides targeted funding for cancer research, including through its European Fund for Strategic Investments ${ }^{1}$.

The above results are mixed, which has led some to advocate for a stronger reli- ance on the mechanism of action principle and legislative changes to the waiver concept in order to force companies to invest more in the development of paediatric cancer medicines. This could however impact the predictability of the scope of PIP and may lead companies to reconsider the overall product development.

EMA reviewed in 2015 its class waiver decision in light of the mechanism of action principle, thereby limiting its scope. This approach may help to engage with companies that develop anti-cancer medicines. If those companies still want a through a direct application to the Paediatric Committee (via a product specific waiver). The process allows direct discussion to highlight the paediatric potential, despite that waiver application. It will also force companies to contact the committee at an earlier stage in the development to get certainty ments under the Regulation. The effects of this class waiver review have yet to be seen as the three-year transitional period has yet to lapse, but the approach may allow for a better buy-in by companies rather than imposed statutory rules.

In addition, it is still not fully understood why companies refrain from reaping the benefits of the Orphan Regulation for paediatric cancers in a similar way that they do for adult cancers. A considerable number of new adult cancer products thrive on the stimulus provided by the Orphan Regulation, while this is not matched for paediatric cancers, albeit all qualify as rare in the sense of the Orphan Regulation.

\section{ADVANCING} PURE PAEDIATRIC DEVELOPMENTS

The positive impact of the Regulation and the change in culture that it has stimulated are most visible in the integration of paediatric development in the overall development of new medicines. It is less obvious in pure paediatric de velopments, which are not a derivative of an adult project, but where a company aims at developing a child-only medcine for a particular paediatric disease.

The available data does not provide sufficient evidence for any firm conclusion Some argue though that for child-only products the PIP process adds an additional layer of complexity for a product that was destined to treat children, potentially prolonging developing timelines While mittee may still provide useful guidance and will ensure a development that covers all relevant paediatric subsets, the impact is less significant compared to adult-based developments. That said, at least in the initial years of the Regulation, companies may have prioritised paediatric projects that are linked to an adult development over paediatric-only projects to ensure its timely completion While this could change over time, es pecially for rare paediatric diseases it seems necessary to better understand the combined impact of the Orphan and Paediatric Regulation and how they and Paedlatric Regulation and how they those statutory instruments in paediatric only diseases.

There is one category of paediatric only developments where the Regulation tries to generate specific interest but has so far failed. It introduced the concept of a paediatric use marketing authorisation (PUMA) The main goal of the PUMA concept (Article 30) is to stimulate research in existing compounds that are off-patent and/or to help transform known off-label use into authorised use that is safer and better framed through the marketing authorisation. Once approved, the PUMA provides the manufacturer with a ten-year which generic copies cannot be placed on the market.

To date only three PUMAs have been granted. This is clearly below expected levels, given that ear-marked EU funding from the FP7 programme has beending from the provided for several years for off-patent medicines. While EMA agreed more than 20 PIPs with a view to submitting a PUMA, it remains uncertain how many will ever be completed and lead to the commercialisation of a new product.

In an attempt to create additional interest, the Commission and EMA clarified in 2014 that a PIP for a PUMA does not have to necessarily cover all age groups, but the impact has so far been limited. While this may allow companies to focus research on the most prevalent paediatric subsets, it risks further reducing the target population and potential revenues.

The PUMA concept struggles with similar issues like any scheme mean to encourage companies to invest in additional research for known compounds that have been on the market for a long time (repurposing). Medicine for a long time (repu a will not necessarily prevent physicians from continuing to use competitor products with the same active ingredient but authorised for other indications offlabel, at lower costs, nor substitution for cheaper forms at the level of pharmacies Moreover, national health care payers are generally hesitant to agree a premium price for such products.

Given the current limited number of granted PUMAs it is neither possible to check whether those risks are substantiated nor the economic value of the PUMA reward. While the available data 
shows that the products authorised through PUMAs have received positive reimbursement decisions in several Member States and represent good business cases, it may simply be the exception to the rule, partly supported by the specificities of the products ed by the specifities of the products

This shows that the commercial success of a PUMA is influenced by complex factors that can be hardly addressed at EU level. They concern downstream decision-making at national level, which is outside the scope of EU law. Legislative incentives canof EU law. Legislative incentives cannot compensate for economic success. There have been suggestions that a PUMA might be effective where a childspecific formulation or dosage form is required, but while this hypothesis is valid in theory, experience shows that the PUMA label does not fully exclude physicians to prescribe nonchild-adapted products.

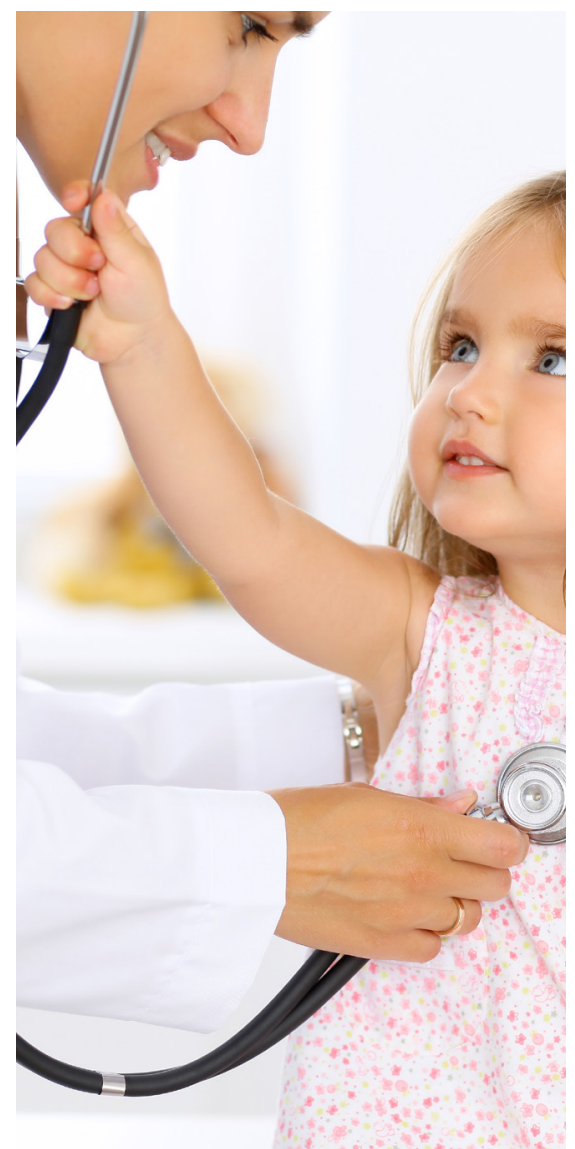

THE COSTS OF PAEDIATRIC MEDICINES

The Regulation places an additional burden on pharmaceutical companies by asking them to carry out paediatric research, which they might not have undertaken otherwise. It requires additional investment and compliance monitoring. The Regulation however, links this obligation with a reward system in order to allow companies to recuperate the additional upfront costs incurred as a result of it through prolonged protection periods. In this regard, the EU system differs from the US system, where paediatric requirements imposed by US Food and Drug Administration (FDA) do not attract a reward, with the exception of those where a company voluntarily engages in additional research following a corresponding 'Written Request' by US FDA.

The reward becomes available once the PIP is completed and its results are reflected in a corresponding marketing authorisation The company is entitled to the reward even if studies finally fall to support a paediatric use of the compound, as it is meant to compensate for the research as such, not a particular outcome. The Regulation differentiates between two main rewards, the SPC reward and the orphan reward. They are mutually exclusive and serve different purposes, but have both the effect of delaying the market entry of competitor products. Hence, the additional revenue provided by the rewards are finally covered by national health care payers and/or patients, as society does not benefit from increased competition and lower prices for the duration of the exclusivity extension.
Under Article 36 of the Regulation, the company may obtain a six-month extension of the duration a six-month extenan autonomous sui of the SPC. SPCs are the existence of a basic patent. They compensate a patent holder for the lengthy periods for obtaining marketing authoriperiods for obtaing marketing authorsation during which the patent owner cannot commercially exploit the patent. The SPC therefore provides a patent right-like position for a variable duration (from zero to a maximum of five years). It is this period that will be prolonged through the SPC reward or that may be turned positive if it was previously negative ${ }^{12}$. It is interesting to note that the legislature interesting to note that the Iegislature chose an extenal reward system linked to the patent status of a product over the pharmaceutical-specific reward system of regulatory data protection.

The orphan reward (Article 37) consists of a two-year extension of the orphan market exclusivity period i.e. up to One of the reasons for intoducing an One of the reasons for introducing an orphan-specific reward was that, when the legal proposal for the Regulation was discussed, the majority of orphan designated products were off-patent. It was therefore felt appropriate to provide for an alternative reward in order to ensure that manufacturers of orphan medicina products may also have access to compensation.

The system of the Regulation is built on the assumption that products falling within the PIP requirement should be eligible for the reward, once the paediatric development is completed. However, in reality not all companies were able to obtain a reward. Figures sugest that up to now only $55 \%$ of the compla benefited from a reward. Most of them took the form of a prolongation of the SPC. In a few cases the market exclusivity period of an orphan medicinal product was granted. While it is expected that over time the proportion of products that benefit from the reward will incease, as beneft from the reward will increase, as companies start to plan better and earlie their paediatric research, it is unlikely that the success rate will ever reach $100 \%$ 

certificate reward

The SPC prolongation is often considered as the most precious reward. Until the end of 2016 more than 40 medicines benefited from the SPC reward and companies applied for respective certificates at national level. The number of SPC prolongations granted in the last 10 years (more than 500) shows that companies regularly receive the reward from the national patent office to which they apply. This points to a functioning reward system.

At the same time, the use of an external reward system linked to another legal instrument, leads to complications and inefficiencies. For example, SPCs are national titles, which means that extensions must be obtained from the national patent office in each Member State in which an SPC exists, and thus considered an $\mathrm{SPC}$ exists, and thus considered by

Moreover, filing for the SPC extension must happen two years before the expiry of the certificate. In some cases, this resulted in companies missing out of the reward as they failed to complete the PIP on time. On the other hand this deadline stimulates companies to speed up the completion of paediatric research and ensures that generic competitors learn sufficiently in advance about any prolongation of the protection period that may impact the market launch of generic copies.

The SPC Regulation is currently subject to an evaluation initiated by the Commission to assess the usefulness of the instrument ${ }^{13}$. Its results and the consequences for the future of the SPC system are not yet known, but expected in the coming months. Any modernisation or recalibration may address some inefficiencies of the SPC system, but may also ciencies of have a direct impact on the functioning of the paediatric reward system and thus on the Regulation itself. It is therefore important to take the results of that evaluation into account in any policy decision about the Regulation.
The monetary value of the SPC rewards depends largely on the overall revenue that a particular product brings in during the period in which it is protected by an SPC. Generic competition will be delayed for the entire product (including the adult use), securing the marketing authorisation holder an additional period of premium revenues. Historically, this period corresponds with the peak in sales. However, new market trends may lead to a decrease in revenue return times. The market position of a product may be diminished over time by the market entry of new innovative products in the same therapeutic class.

To estimate the economic benefit to companies deriving from the reward it is first necessary to establish the regulatory costs incurred by companies to comply with a PIP. Based on an external study ordered by the Commission ${ }^{14}$ the total costs of the total costs of the Regulation for the whole industry is estimated to be EUR 2.1 billion per year. This figure derives from an extrapolation based on 85 real PIPs. The total $R \& D$ costs on average amount to EUR 18.9 million per PIP, with each plan including an average of three clinical studies. On top of this, companies incur overhead costs of around EUR 720000 in relation to filing of the initial submission of a PIP and for subsequent modifications.

While those averages build on a relatively robust sample size, risks of over- or underestimation cannot be fully excludd. Moreover, estimations based d. Moreover estimations based on averages means that there are deviations, especially on the costs for clinical trials (phase II and phase III), which account for the largest portion of R\&D costs ${ }^{15}$. Nevertheless, those figures suggest that the additional costs borne by industry as a consequence of the Regulation only lead in the total costs of medicine development.

To compare those costs with the value of the SPC reward eight medicinal products have been specifically analysed. This selection includes products that received
SPC extensions and lost their protection before the end of 2014. The sample size is naturally quite small, as only a fraction of products with completed PIPs have yet lost exclusivity rights and hence, provide data on the impact on such loss on revenues. While the figures for those products may need to be interpreted with some caution, given that companies may have prioritised in the early years products with the highest estimated return of investment resulting from the SPC prolongation, they provide some interesting insight in the economic value of the reward by comparing the actual revenues with the SPC extension revenues without such extension.

The data shows that the price drop of branded products often starts in the first quarter after the loss of exclusivity, but limited in scale (up to $20 \%$ ), before decreasing further. There are significant differences between products and countries, most likely linked to the competitiveness of the particular therapeutic market and/ or national policies to stimulate generic substitution, leading to a high variation of the economic value of the SPC extension as a percentage of the total revenue (between 10 and $93 \%$ ). Overall, the adjusted economic value of the SPC for the eight products concerned amount to EUR 926 million, with revenues especially geared towards some blockbuste products included in the sample size.

While this figure may be compared with the average R\&D costs per PIP (18.9 million), a more granular approach may focus on a product based benefit-cost ratio of the eight developments. This means comparing the estimated benefits for society and child health resulting from the enforced paediatric development with the costs to society from the extra monopoly rent obtained by the company through the reward system.

Such comparison is exploratory in nature as it has to put a monetary value on the
positive impact in terms of improved positive impact in terms of improved
treatment for children and a reduction treatment for children and a reduction of off-label use and also the potential model developed as part of the economic study, two out of the eight products show a strongly favourable benefit-cost ratio for health systems when calculated over a 10-year period, i.e. the benefits for society and health in monetary terms outweigh the additional costs due to the extra monopoly rent. All other products have a negative benefit-cost ratio over 10 years, especially those for which the completion of the PIP did not result in a new paediatric indication. While it is still useful to know with certainty that an adult product should not be used in children, the economic value of such information is much smaller compared to products, which provide new treatment alternatives for paediatric patients.

Those product-based results may how ever need to be adjusted with those products, which had to comply with the PIP obligation, but were not able to (around $45 \%$ ). They resulted in valuable paediatric information becoming available without society contributing to the incurred costs through additional monopoly rents. If those products are parts of the equation, results improve but the benefit-cost ratio is still negative.

In addition, the Regulation may generate economic spill-over effects due to additional R\&D investment towards new and improved medicines that triggers furthe investment and contributes to the creation of jobs, growth and innovative activ ity across sectors. A more conservative estimated rate of return from an annual R\&D could after 10 years, yield a total societal return of around EUR 6 billion ${ }^{16}$ This estimated societal return is significantly higher than the economic value of the SPC extension, suggesting that in monetary terms, the benefits of the Regulation for society outweigh the costs of the additional monopoly rent. 
So far seven products obtained the orphan reward of two additional years of market exclusivity, with the first product in 2014. However, in some instances companies voluntarily waived the orphan compan designation in order to make the product eligible for the SPC reward. This may be explained by the fact that the SPC reward protects the entire product family of a specific compound across different therapeutic indications, while the orphan reward is limited to protecting the orphan use. Therefore, where medicines have both common and rar have both common and rare conditions, revenues from a 6 month SPC prolongation might be higher than from an additional two years market exclusivity in the orphan condition.

A contributing factor may be that more and more of newly authorised orphan ancolucts are on-patent (curredly mon products a than $90 \%$ ), which is a positive news, as it shows that the system provided by the
Orphan Regulation attracts innovative Orphan Regulation attracts innovative products based on new research. On the other hand, it points to a weakness of the orphan reward, which is mainly geared towards off-patent products and has no built-in flexibility to allow companies to keep the orphan status of the product, while opting for the SPC reward.

At this stage and without further studies, it is not possible to estimate the economic value of the orphan reward, based on a similar sample size as for the SPC reward, given that most of the products are still under protection. Therefore, it is not possible to analyse the actual impact of the loss of exclusivity on revenues. There is for example no guarantee that generics will enter the market in the same speed as for non-orphan products or at all in view of the rarity of the disease and the limited size of the relevant market Neverthe mack night be used for estimating model might be used for estimating the economic value as the approach used for the calculation of the SPC reward, with the main difference that the delay is two years instead of six months.

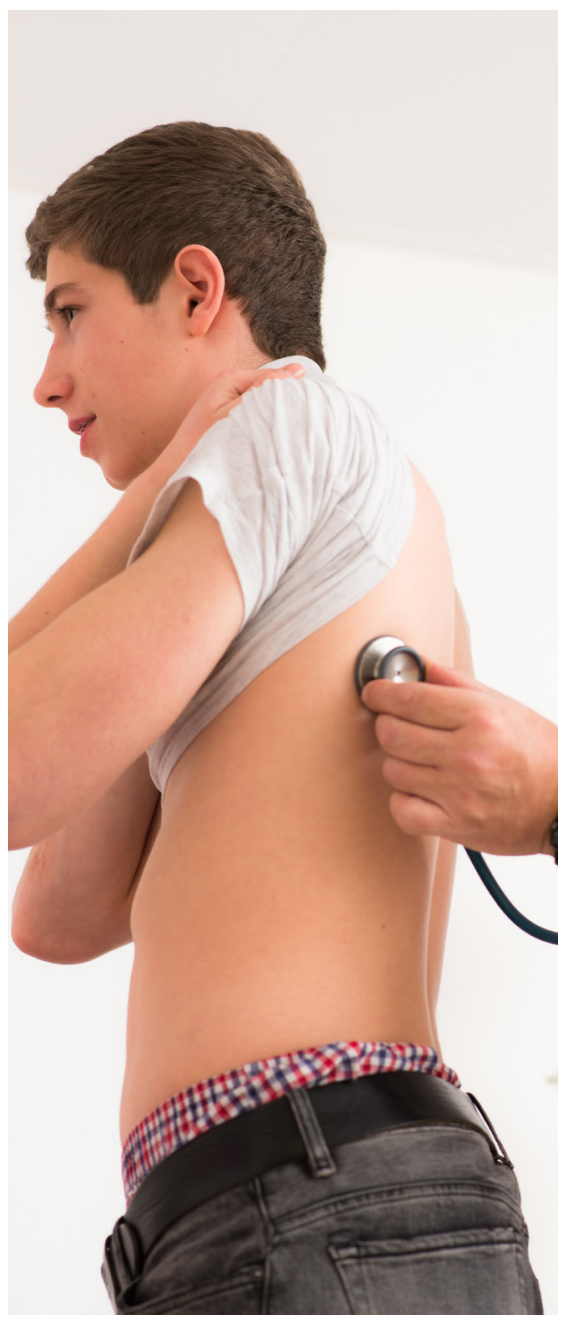
particu scientific committees or advisory working parties. Continuous improvement activities are ongoing to enable such collaboration.

To increase cooperation across regions, a discussion forum to regularly exchange information mainly via teleconferences ('paediatric cluster') was formed in 2007, including members of the US FDA and EMA. The cluster has since been joined by the Pharmaceuticals and Medical Devices Agency (PMDA) Japan, Health Canada, and the Australian Therapeutic Goods Administration (TGA) as an observer In 2013 tration (TGA) EMA and its US counterpart launched so-called 'common commentaries' on paediatric development plans that have been submitted to both the EMA and FDA and that are therefore being reviewed by both agencies. While informal and non-binding, these commentaries and discussions between the two agenand have helped to align views and cies have helped to align views and to avoid contradictory requirements on the paediatric development programme.

However, it remains a challenge for EMA and its Paediatric Committee, as well as for companies, to consider key aspects of medicine development when certain info certain inormation is not yet known and when discussions are still based on assumptions and scarce data. This is true especially as one of the objec tives of paediatric development plans is to create legal certainty on regulatory authorities' expectations towards companies. On the other hand, only early planning makes it possible for paediplanning makes it possible for paediatric development to be seamlessly integrated into overall product development instead of being an afterthought. In principle, it should also lead to more (cost-)efficient R\&D, as it allows for example to consider integrating paediatric patients (e.g. adolescents) in adult trials and into early formulation development and into ealy formulation development planning, therefore reducing overall development costs. development.

Ensuring product discussion and know ledge exchange across the various committees and working parties w remits is an essential the EMA's coordination function. area of paediatric development this of the Paediatric Committee with other 
8.

MORE

CLINICAL

TRIALS WITH CHILDREN

The Regulation aims to ensure that evidence of the quality, safety and efficacy of medicinal products is generated before a product is used by children. This means more clinical research carried out in children prior to the authorisation of medicines. Available figures show a notable increase. The proportion of clinical trials in the European clinical trial database EudraCT that include children has increased $50 \%$ in 2007-2016 from $825 \%$ to $124 \%$ Moreover, 2016 from $825 \%$ to neglected paediatric subpopulations has risen considerably. Prior to the Regulation research with neonates was almost nonexistent in medicine development.

Generally speaking, EU legislation is well equipped to ensure that paediatric research is scientifically valid and ethically sound. These aspects are considered not only by EMA's Paediatric Committee in its assessment of PIPs, but also by national ethics committees and regulatory authorities that are responsible for authorising individual clinical trials.

The Regulation has fostered expert discussion about the optimal design of paediatric trials. This includes initiatives related to the exchange of good practices, and development of new scientific guidelines. A contributing factor has been the creation of a network of research networks at the EMA (Enpr-EMA) ${ }^{18}$, which due to its successful work now expanded beyond Erope, with the registration of American, Canadian and Japanese national and multi speciality networks.

The further development of innovative trial concepts as well as modelling and simulation strategies to reduce the number of necessary study participants, were also stimulated. Additionally, the Regulation brought attention to the debate about the role that children should play in research decisions. Initiatives range from the creation of young people advisory groups to ory groups to discussion of appropriate information about clinical studies for patients and parents, to practical issues, such as consent forms.

Still paediatric trials pose particular challenges. For example, recruitment difficulties frequently lead to delays in conducting and completing them. Paediatric trials also tend to be multi-centre Paediatric tials also tend to be multi-centre trials, sometimes with just a few patients per site, which can create operational challenges, including with maintaining the necessary staff and expertise onsite. To support paediatric clinical trial infrastructure further, the EU-financed private-public partnership the 'innovative end of 2016 a project to create a sustainable PanMoreover, with its recent initiative to establish European Reference Networks, ${ }^{20}$ the Commission supports virtual networks involving healthcare providers across Europe to tackle complex or rare diseases and conditions that require highly specialised treatment and a concentration of knowledge and resources. Some of the thematic networks included in the project focus specifically on rare paediatric diseases. They will foster cooperation and pave the way for additional clinical research which might previously not have been feasible.

Overall, the Regulation has boosted paediatric research. It is however, recognised that such research is geared towards product development. For some diseases or therapeutic areas, a good understanding of the underlying disease is diseases themselves $u$ therefore be beneficial to enable and inform appropriate product development. This cannot be guaranteed through the Regulation, but requires additional efforts and funding from public and private sources.
9.

\section{FUTURE}

CHALLENGES

The way pharmaceuticals are developed may change over time due to scientific advances, technological developments and changing business models. Recent trends include the stratified development of medicines or the concept of personalised medicine, which aims to optimise the use of to patients' individual genes to ensure that they will be tuly responsive to treatments. They may also sponsive to treatments. They may also see the increased market entry of technology firms to support therapies through technology enabled patient support and services.

While most of these new development paradigms seem perfectly compatible with the mechanism introduced by the Regulation, they may influence the way companies decide on investment priorities and design clinical trials. In the short term those trends are unlikely to affect the Regulation as the number of new medicines in late stage pipeline is historically large, wit tive substance forecast to be launched per year until 2021. However, the PIP process needs to provide the necessary flexibility to accommodate those trends, while at the same time ensuring that children fully benefit from those emerging concepts such as personalised medicine.

On a more granular level, it also needs to be taken into account that the implementation of the Regulation presupposes a significant investment of resources no only from $E M A^{21}$, but also by Membe States, by appointing members to the PDCO and contributing to the assessment of paediatric investigation plans or historical or new paediatric trial results submitted by companies. The Regulation states that applicants can avail of these procedures without incurring any fees, which is part of the incentives to enable paediatric development. While there is no evidence that the absence of fees had so far a negative impact on the quality of the assessment, the long-term impact on the proper $f u n$, the long unknown. In its ongoing evaluation of the EMA fee system the Commission will also verify the costs of assessing PIPs.

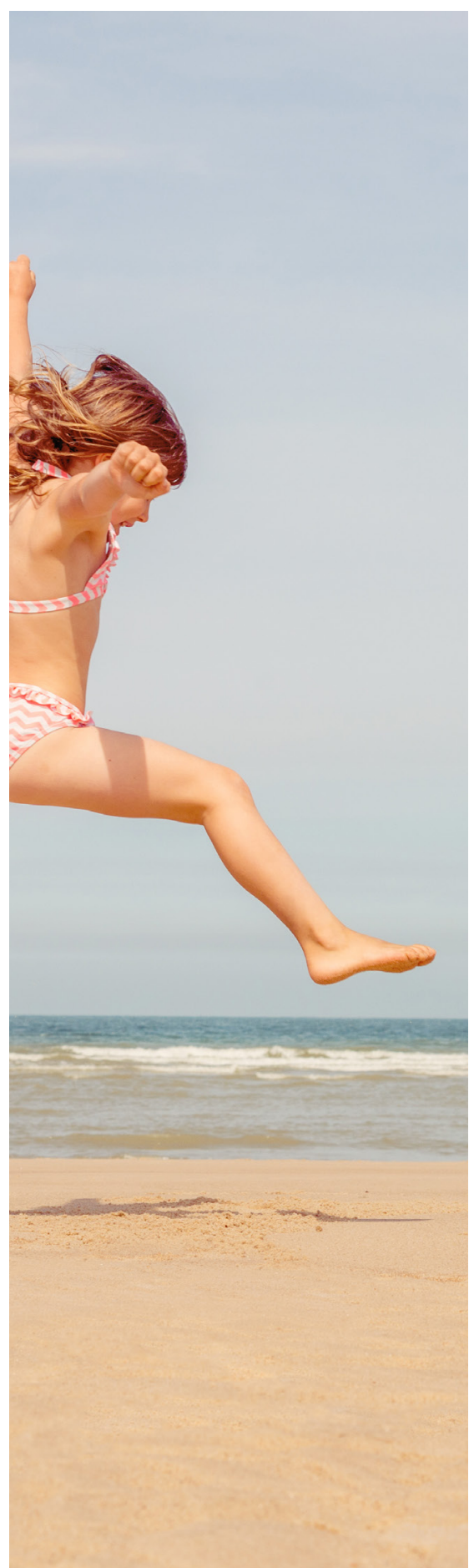


CONCLUSION 
10. CONCLUSION

The Paediatric Regulation had a considerable impact on the development of paediatric medicines in the EU. It ensured that paediatric medicine development became an integral part of the overall development of medicines. This result wo without specific logislation and underlines its continued relevance. Moreover, measures taken to improve its implementation have over time strengthened its effectiveness.

In economic terms, the Regulation provides overall positive result vides overall $p$ cioeconomic perspective demonstrating the appropriateness of this direct investment in improving the availability of paediatric medicines. The combination of obligations and rewards seems effective to shift focus to paediatric product development Still the use of rewards was limited to 55 \%o of the comple PIPs and there are instances of overPIPs and there are instances of over- or under compensation pointing to certain limitations of the current system. Additionally, the PUMA concept with its specific reward has failed to deliver.

The increase in paediatric research and the number of new products with specific paediatric indications is encouraging and will ensure that over time the offlabel use of adult medicines in the paediatric population will decrease. Those positive results do however not evenly spread among all therapeutic areas, but concentrate in some, often linked to research priorities in adults rather th children.

This shows that the Regulation works best in areas where the needs of adult and paediatric patients overlap. Especially, in diseases that are rare and/or unique to children and which in many cases are equally supported through the orphan legislation major therapeutic advances often failed to materialise yet.
Why this is the case and why the orphan reward is in some instances not able to drive paediatric development in a similar way than adult orphan development requires further scrutiny.

Therefore and before proposing any amendments, the Commission intends to take a closer look at the combined effects of the Orphan and Paediatric Regulation through a joined evaluation of those two legal instruments aimed at supporting medicine development in subpopulations of particular need. Given that weaknesses identified in this report that wate to partic often relate to paediatic diseases that qualify as orphan condition, only such combined effort will guarantee to adjust the right parameters, if required.

This report marks not the end, but an essential intermediate step in the debate on a joint vision about the future for paediatric and orphan medicines. The further evaluation supporting this process aims at providing results by 2019 so to allow the next Commission to take informed decision about possible policy options. It will also allow the forthcoming results of the SPC evaluation for the future of the Paediatric Regulation to be taken into account.

In the meantime, the Commission is committed to a positive agenda of concrete actions in order to streamline the current application and implementation together with EMA wherever needed. This includes:

\section{- providing additional transparency}

of new products authorised with paediatric indications;

- analysing the experience with use of deferrals and consider changes in practice to ensure speedier completion of PIPs;

- revisiting processes and expectations in the context of handling of applications for PIPs and if necessary adapt the corresponding Commission guideline;

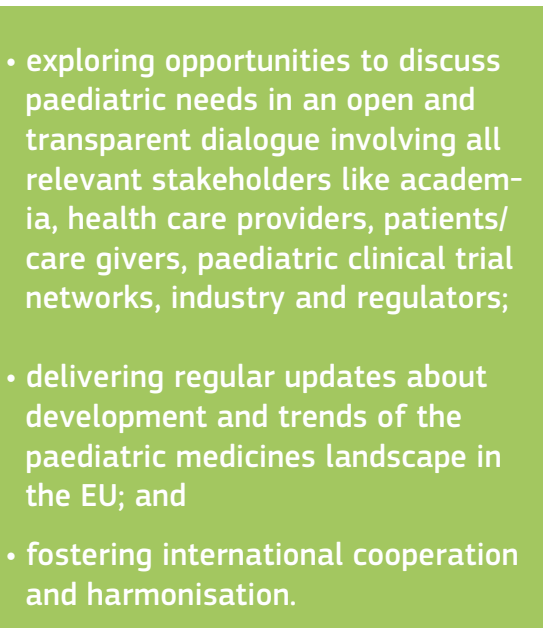

Additionally, it will further support highquality healthcare and research for children through projects such as the Euronetworks, which connect health care providers and centres of expertise. Those networks have the potential of significantly improving access to diagnosis and treatment in the short term and to make a difference in terms of child health.

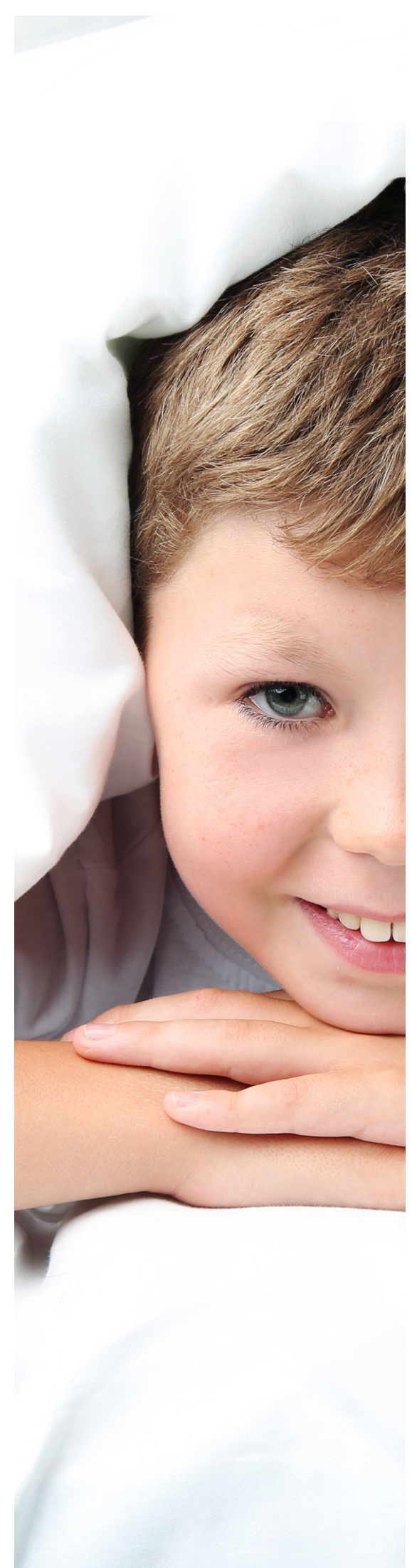




\section{More paediatric trials}

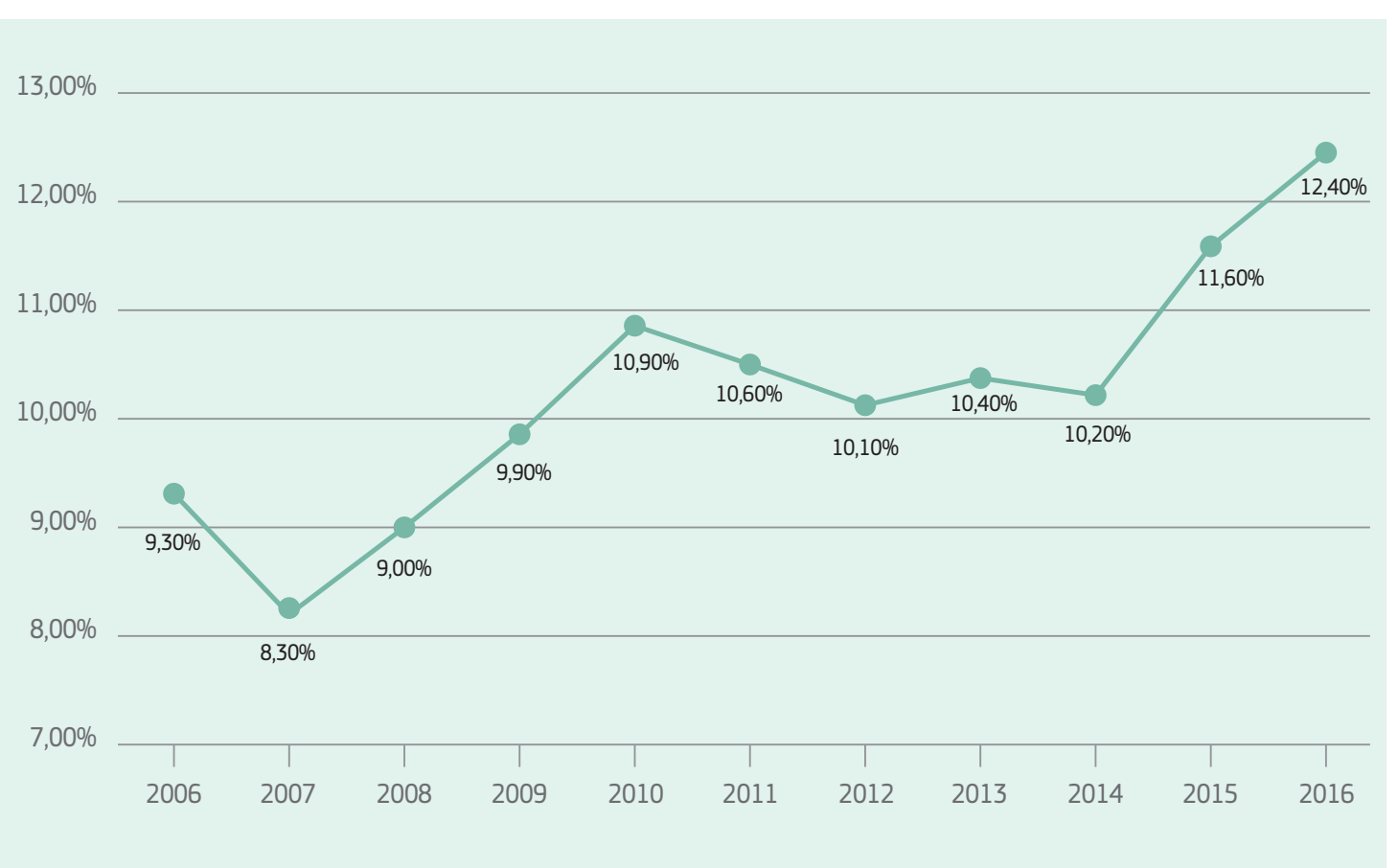

Source: EudraCT database

\section{More authorised medicines}

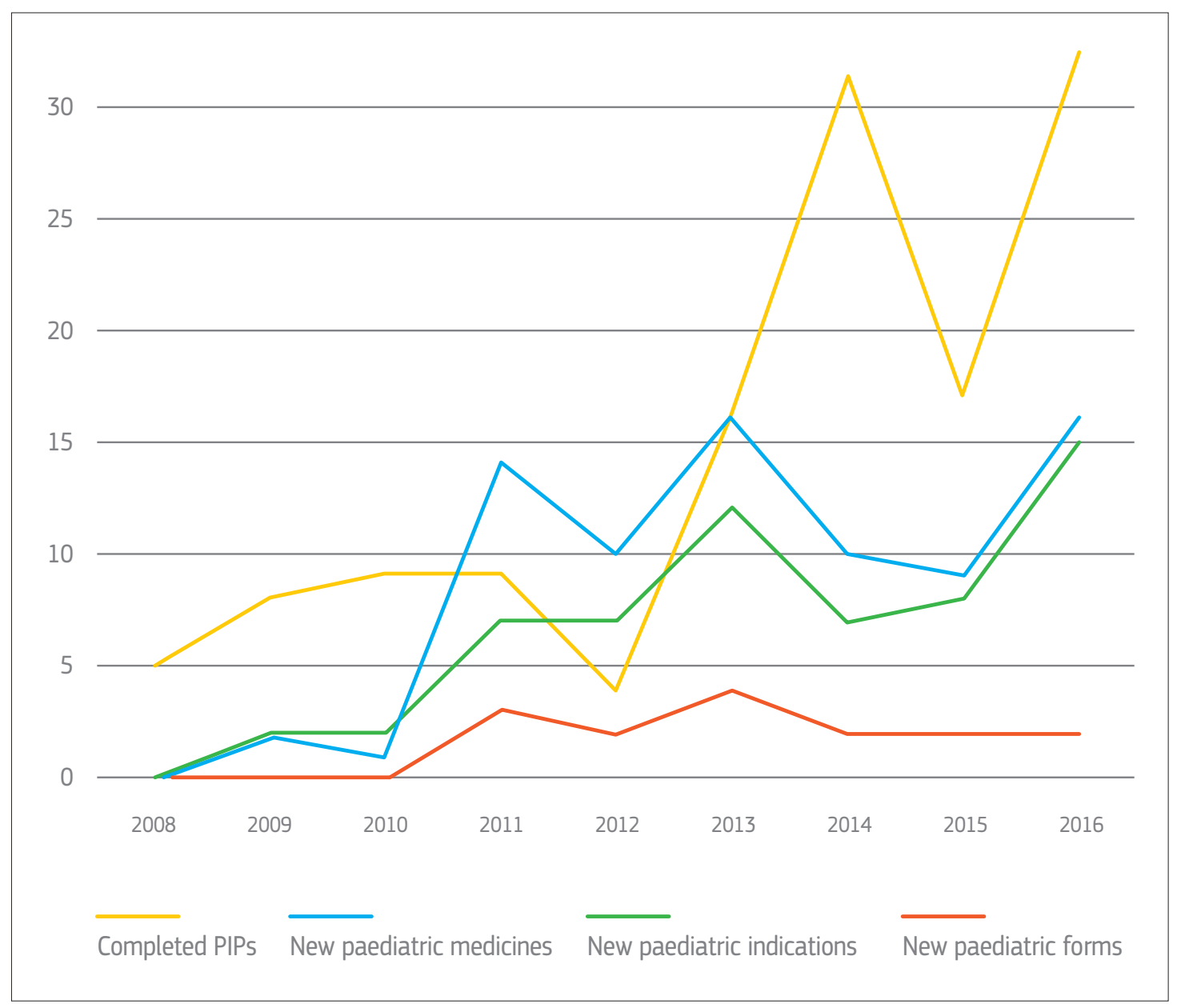

Source: EMA databases (only centrally authorised medicinal products). 


\section{More paediatric information}

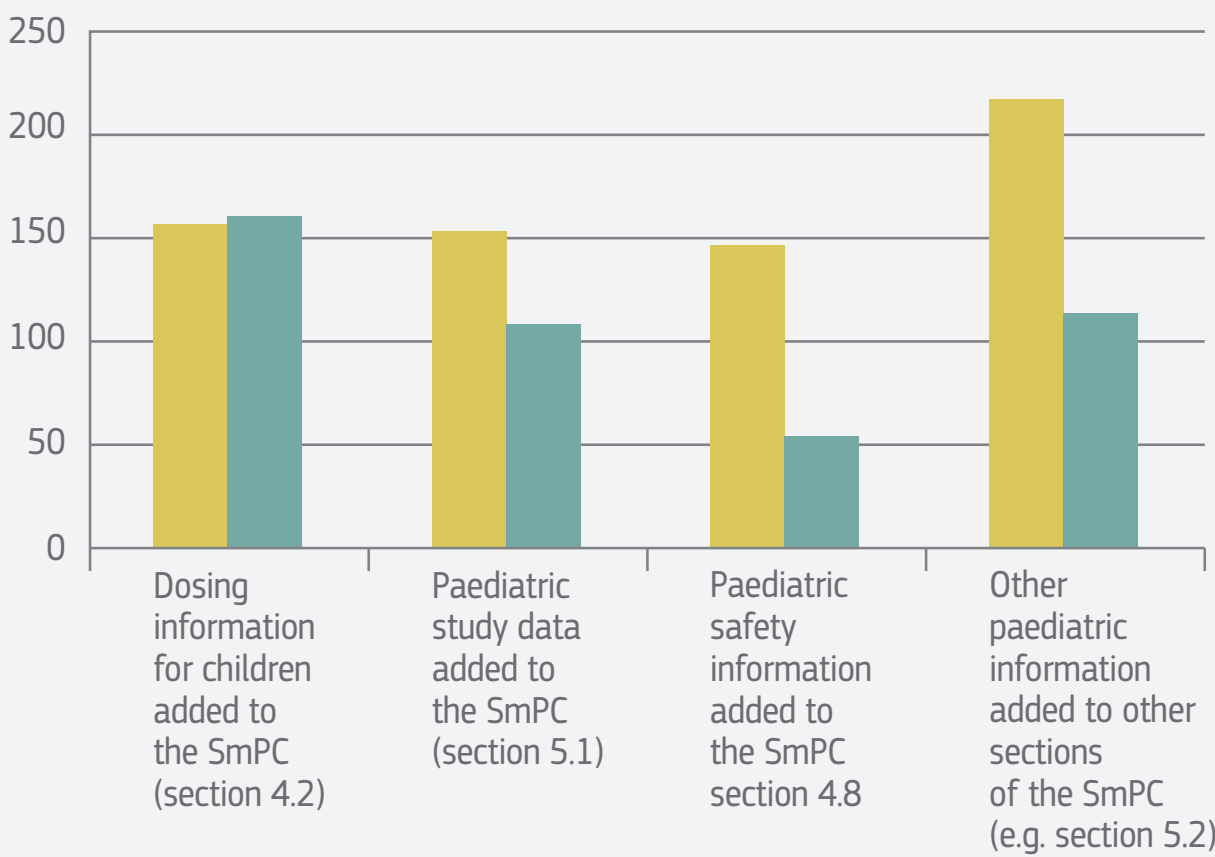

SmPC: Summary of product characteristics
Increased information
on centrally authorised
on nationally authorised
medicines for paediatric use
medicines for paediatric use

\section{Therapeutic areas addressed by the paediatric investigation plans (2007-2015)}

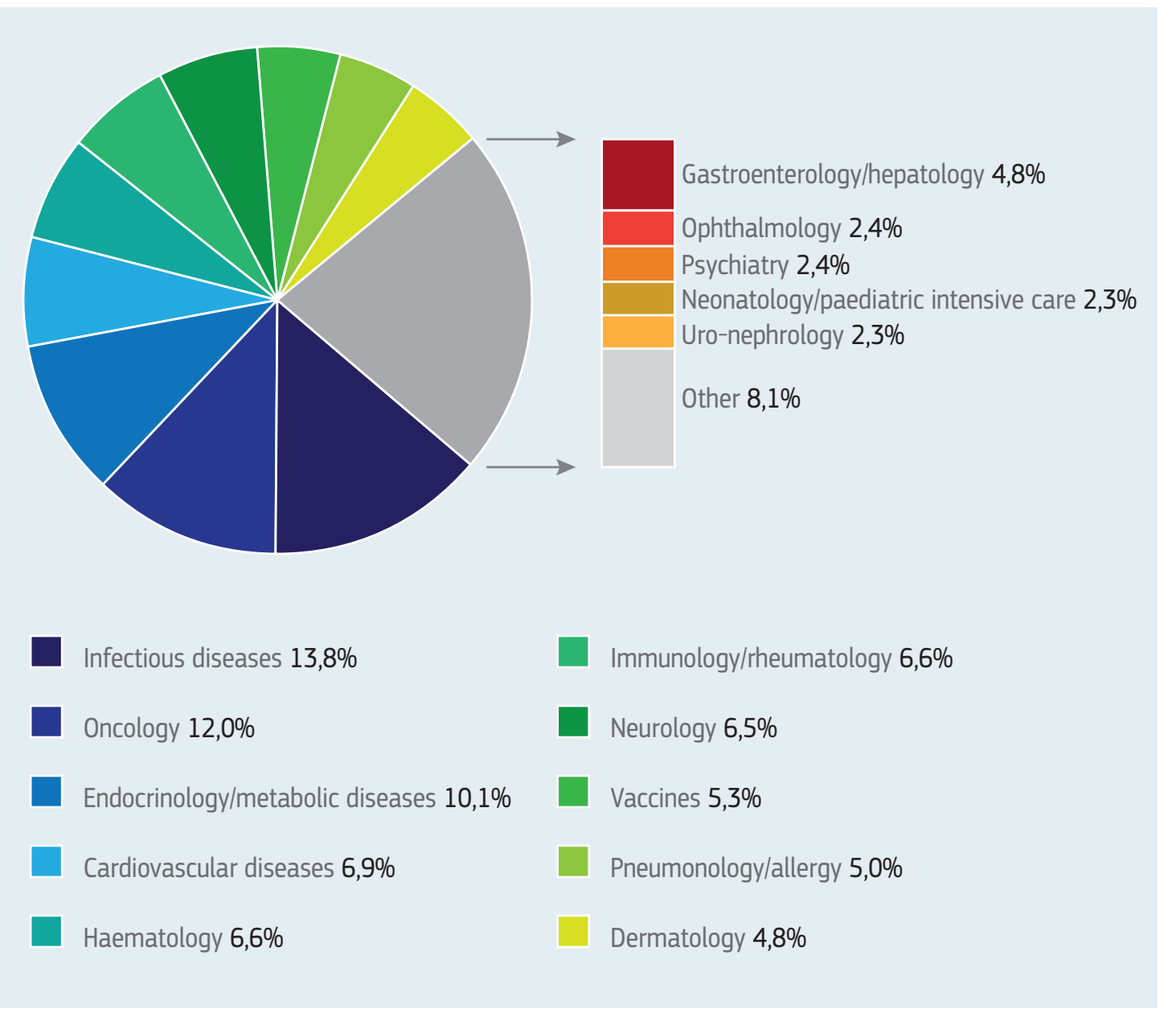

Source: EMA database (PedRA)

$\square$ Infectious dise
Oncology 12,
Endocrinology
Cardiovascula
Haematology


NOTES 
